\title{
Coupling Analyses between Generality of Product Design and Product Image System
}

\author{
Yihui Wang \\ Urban Vocational College of Sichuan, Sichuan Chengdu, 610100
}

Keywords: versatility; product image; coupling

Abstract: The versatility of industrial design products is a large and systematic research area. The product image system includes three parts: basic system, product application basic system and product application promotion system. In the product design and development process, it is necessary to meet the versatility and also identify the product image. This is a contradictory relationship. The effective coupling between the two can make the product more humanized to be in line with the market, which will be the most important design and market reference for industrial enterprises to provide industrial products in the future.

\section{PIS and product design versatility}

The versatility of product design is a complex and multi-angle category, including emotional cognition, friendly interface, human-machine relationship, sensory experience and many other factors. From the design point of view, it means that the design and environmental considerations of the product are the largest. It is possible to create design activities for all users to create a more inclusive environment and products that enable universal care for product users. A design concept that reflects equality, love and caring for everyone. Through the designer's inductive research on product versatility, it can be divided into fair use, flexible and convenient, simple and intuitive image, accurate information transmission, error correction device, labor-saving use, proportional coordination of size space, economy Durable, high quality and beautiful, no harm to the human body and the environment, the versatility of product design is not static, it will be more perfect with the development of society and the changes of human history.

PIS is the abbreviation of English Product Identity System, which means the product image unified identification system [1]. It is a market-oriented image system that is more suitable for global market operations and the needs of domestic companies. There is an image metaphor. If the CIS is regarded as an aircraft carrier, then the product image PIS is an efficient and flexible torpedo speedboat. PIS contains specific content such as product name, logo, culture, color, and packaging. PIS pays more attention to the performance of products on the terminal. In terms of form, it is more detailed and classified in more detail. From the perspective of communication, the products can be displayed in front of consumers in a series and overall style with visual maximization. Thus, the potential consumption is changed to actual consumption, and the accidental purchase is made for long-term purchase. In terms of investment, the market can be quickly started with a small investment and a synergy. Popularly speaking, product image recognition is a purposeful shape, 
which can be called the DNA of a product. It can also be called a product that is a weapon of the king. With the diminishing technical differences, the establishment of a unique and effective product image recognition system has a significant effect on improving product image and market competitiveness [2].

The two seem to be opposite, but they contain each other. In the end, the product is better able to meet the ultimate goal of consumer use. The contradictory image has a common purpose. Therefore, this is a contradictory and antagonistic relationship that can be reconciled and optimized. Coupling, as a noun, is commonly used in communication engineering. This term is now used in the relationship between the two. In a nutshell, it is a measure of the relationship between the two depending on each other, in order to show the paradigm relationship of interdependence.

\section{Paired coupling analysis}

According to the PIS modular research, it can be divided into positioning strategy, real analysis, visual system, monopoly system, regular marketing, and network marketing. According to the principle of pairing research, the six modules in the PIS can be organically combined with the product design versatility 10 standards to achieve the coupling effect.

\subsection{Positioning strategy and fair and flexible use of design rules}

The positioning strategy in PIS points the direction to the original concept of the product and is an important factor in the success or failure of the product. It can not only improve the image of the product, but also make the goods express and convey the feelings, improve the loyalty of the products, and make the products unique in the fierce market competition.

Fairness is the design that is available and suitable for people of different abilities. For example, the shape of the drum washing machine has always been square, most of them adopt the front door opening method, and some adopt the top door opening method for taking care of small space users. Both of these methods have their limitations, and users of different heights have inconveniences when using them. Since the birth of the drum washing machine, this shortcoming has been accompanied by users. In recent years, some manufacturers have been concentrating on developing their concept of "universal design" and designing inclined drum washing machines to take care of the operation modes and habits of various users. The front door of the drum washing machine was creatively tilted by 30 degrees, and the door was opened obliquely, and the center axis of the inner drum was also inclined by 30 degrees from the horizontal direction. This design allows anyone to use the washing machine equally, safely and conveniently under any circumstances.

Flexibility is the design of a user who can flexibly adjust to a wide range of wishes and abilities. For example, the telescopic bed in the home design, the movable patchwork bookshelf, this is the embodiment of flexibility, allowing consumers to adjust the control of the product, reflecting the sense of accomplishment.

The combination of positioning strategy and flexible and fair design rules is mainly reflected in: creating a warm product culture. A product is easy to use, suitable for both young and old, and can flexibly adjust the state and size. This is a very interesting phenomenon. I believe many consumers will not let go of him, and more will fall into love. Industrial design is a combination of art and science. Products are the crystallization of science and art, making products beautiful. It is the focus of industrial design, but the fair and flexible design rules make people enjoy the beauty. This is also the positioning strategy. The value of a product is related to the survival value of the product itself. No one wants to buy something that is troublesome and inconvenient to use. In reality, most of the products are to meet the needs of the public. Since the products are for the public, the PIS positioning requires designers to analyze and control, fair and flexible design principles are 
particularly important. The use and practicality of this product is the best interpretation of product positioning. The appearance of the product is of course important, but in the era of minimalism and practicality, intimate functional design is the most critical factor. The so-called "small places see the taste", the reasonable evaluation of the product features, will make the product more widely applicable, the people involved are more subtle, so that the value of the product is greatly improved and accepted by consumers.

\subsection{Real-state analysis and labor-saving, economical and durable design rules}

Product real-time analysis is one of the important factors to measure whether a product occupies the market. From a design perspective, it refers only to a state in which the product is in space or in use. The effort is that the design should be used effectively and comfortably to minimize fatigue. Economical and durable to meet the cheap and durable psychology of most people, improve the life of the product.

The product is all-encompassing, labor-saving and economical and durable. In fact, it has too many functions to be ignored. The labor-saving design is often very good, because consumers can immediately impress it after using it. If it is equipped with economical and durable effects, then this product is definitely easy to be accepted by the market and quickly occupy market share. Through the online search for keywords "saving effort", we can see a lot of questions and explanations about the various industries and products in all directions, which just gives the designer a question, that is, most of the products on the market may not have considered the factor of labor saving. Therefore, if you consider how to save effort at the beginning of the design, then consumers will not be questioned about the use of the product. Economical and durable, we often call it "price/performance ratio", which is also a crucial factor in determining the success or failure of a product. Through the scientific combination of materials and processes, we can strive to achieve the best price/performance ratio. In the actual state of the product, there are requirements for technological innovation and core values. The use of labor saving and economic durability is their extension. Therefore, the analysis, research, and general design rules at the beginning of the design can often add a lot to the actual state of the product.

\subsection{Vision system with simple and intuitive, accurate information transmission, size and space coordination design rules}

Visual performance is a more intuitive cognitive method, often the perception of first impressions. Simple and intuitive products make it easy for users to understand regardless of their cognitive ability or concentration during use; the information is accurately conveyed so that the product can be effective regardless of the conditions of the environment and the level of user perception. The necessary information is conveyed to the user, and the reasonable design interface can bring the user a feeling of ease and pleasure and a sense of success. On the contrary, due to the failure of the interface design, the user is frustrated, and the practical and powerful functions may be in the user. The fear and abandonment are lost [3]; the size space coordination is designed to provide the appropriate scale and space to adapt to different users' body size, posture or mobility, so that users can access, reach, manipulate and use. These can leave a clear and rational understanding of the product users. If combined with visual perception, this will make the product more understandable and acceptable. The philosophical existence is reasonable, but from the designer's point of view, the existence is unreasonable. Because any product will be flawed, because of the influence of the environment, users, and social culture, the reasonable product will show an unreasonable side. Because this is a constantly changing society, a rapidly changing society, cultural integration, and changes in physiological habits often confuse products that were previously 
considered to be very reasonable and very convenient. Because of this, the visual system in PIS will continue to change and will gradually be replaced, and the only constant is "change", simple and intuitive product image, the accurate and friendly interface of information communication and the proportion of design coordination of size and space are constantly changing subtly, which can continuously improve and enhance consumers' perception of products and enhance the product vision system, it is not just about changing the brand, creativity, packaging, image and other factors to support and update the visual system in PIS. Therefore, the research of product image system theory should be combined with practice, through the "theory-practice-theory-" cycle model to explore the rational and perfect product image system theory [4].

\subsection{Marketing monopoly and quality, environmental protection, error correction design rules}

One of the most important modules in PIS is marketing monopoly. In fact, this is only a theoretical point. Back to the product, what we need is the spirit of "hardening the iron itself". As long as the product quality is excellent, in line with market requirements, humanized design is in place, and then with the marketing monopoly system, this is a gold combination.

Excellent quality and aesthetics require good use of the product, the material selection and functional structure are perfectly combined, and the operation is simple and easy to maintain, and the product cost and the price are reasonable; the environmental protection is the product to meet the green design, ecological design and sustainable development concept; The error correction design should be able to minimize the hazards and negative consequences caused by accidental or misoperation. Imagine that products will be directly in face-to-face contact with consumers during the marketing resale process. If there is a low-abuse quality, harmful side effects on the human environment, trouble caused by misuse, etc., this will be a bad product. Because the consumer market we are facing now is not purely pursuing low prices, but constantly pursuing a better life and experience, so marketing monopoly activities must have excellent products as capital to gain a foothold. For example, in the subway station where the bustle is coming, the station and the outbound person often hang the thin chain of the subway card on the neck in order to catch up with the time, so that the card can be quickly swiped. But have you ever thought that if the thin chain is hooked in a hurry and you can't get rid of it, it is easy to cause harm? To this end, there are designers in the subway card chain plus "clip" design, when the pulling force is too strong, it will automatically break, then just pull the clip back and continue to use. This error-correcting design minimizes the adverse consequences of dangerous and inadvertent actions and is a true portrayal of the versatility of product design. Like this kind of product, I believe that it can be quickly popularized in the marketing process without any effort, which greatly reduces the marketing investment, because consumers' perception of such products is based on quality, error correction, environmental protection and other aspects. Rational cognition, speaking with facts, using live experience and simulation is the best marketing monopoly strategy.

\section{Conclusion}

Through the above analysis, we can see that the universality of PIS and product design is not a contradictory relationship. After digging deeper, we can easily find that they are complementary to each other but interfere with each other. The versatility of product design is a kind of rule that is summarized after in-depth study of people's life and habits. It is the perception and experience of life. PIS is only a theoretical study, in the final analysis, it still needs excellent products to support the foundation. The excellent products require designers to constantly study human habits, and constantly explore and pay attention to the versatility of design. In order to make the product more humane and in line with the market, this will be the most important design and market reference for 
industrial enterprises to provide industrial products in the future.

\section{References}

[1] Xu Juanyan, Chen Yingjie. Research on Model Design of Welding Series Based on PIS [J]. Mechanical Design, 2015(1):126.

[2] Feng Qiang, Bai Zhen. Research on Shaanxi Folk Custom Souvenir Design Based on PIS [J]. Packaging Engineering, 2016, 37(22): 148-152.

[3] Zhao Yuting. General Analysis of the Universality of Interface Design for Handheld Mobile Products [J]. Art Science and Technology, 2013, 26(03): 177.

[4] Yan Qun, Xiao Wangqun, et al. Research and discussion on product image system (PIS) theory [J]. China Packaging. 2009(02) 